International Journal of Wireless \& Mobile Networks (IJWMN) Vol. 5, No. 6, December 2013

\title{
A BI-SCHEDULER ALGORITHM FOR FRAME AGGREGATION IN IEEE 802.11N
}

\author{
Vanaja Ramaswamy ${ }^{1}$, Abinaya Sivarasu², Bharghavi Sridharan ${ }^{3}$ and Hamsalekha \\ Venkatesh $^{4}$ \\ 1, 2,3,4 Department Of Computer Science and Engineering, \\ Sri Venkateswara College Of Engineering, \\ Sriperumpudur taluk, Tamilnadu, India
}

\begin{abstract}
IEEE 802.11n mainly aims to provide high throughput, reliability and good security over its other previous standards. The performance of $802.11 \mathrm{n}$ is very effective on the saturated traffic through the use of frame aggregation. But this frame aggregation will not effectively function in all scenarios. The main objective of this paper is to improve the throughput of the wireless LAN through effective frame aggregation using scheduler mechanism. The Bi-Scheduler algorithm proposed in this article aims to segregate frames based on their access categories. The outer scheduler separates delay sensitive applications from the incoming burst of multi-part data and also decides whether to apply Aggregated - MAC Service Data Unit (AMSDU) aggregation technique or to send the data without any aggregation. The inner scheduler schedules the remaining (delay-insensitive, background and best-effort) packets using Aggregated-MAC Protocol Data unit (A-MPDU) aggregation technique.
\end{abstract}

\section{KEYWORDS}

IEEE 802.11n, Frame Aggregation, Scheduler, A-MPDU, A-MSDU.

\section{INTRODUCTION}

IEEE $802.11 \mathrm{n}$ is the most popularly used wireless Local Area Network (LAN) technology today. It is one of the superior standards when compared to its previous standards like IEEE $802.11 \mathrm{a} / \mathrm{b} / \mathrm{g}$ through its ability to provide maximum of $600 \mathrm{Mbps}$ [4] data rate. This is due to the enhancements provided in IEEE 802.11n like Multiple Input Multiple Output (MIMO) and channel bonding [1] in PHY (Physical) layer and other enhancements in Medium Access Control (MAC) layer. The MIMO technology in PHY layer uses multiple antenna elements to send multiple copies of the data there by significantly increasing the data rate. It uses $40 \mathrm{MHz}$ channel for transmission. The MAC layer enhancements are frame aggregation, block acknowledgement, reduced inter frame spacing and allowing transmission in reverse direction. Recently, many $802.11 \mathrm{n}$ based products are popularly used in many user devices. But the performance of these products is not up to the expectation due to various constraints in the real time. The fairness of the IEEE $802.11 \mathrm{n}$ is not very effective in case of unsaturated traffic.

This paper proposes a scheduler for the MAC layer which divides the packet based on Access Category (AC) to provide different QoS services for different category of data. It also decides between whether to use frame aggregation or not based on the urgency of the data. This provides fairness to the traffic for off time traffic.

DOI : 10.5121/ijwmn.2013.5607 


\section{RELATED WORK AND LITERATURE SURVEY 2.1 PHY and MAC layer enhancements}

The important enhancements in IEEE 802.11n which improved the performance of the WLAN are briefed here.

\subsubsection{Multiple Input Multiple Output (MIMO)}

IEEE 802.11n uses MIMO technology to improve the data rate up to $600 \mathrm{Mbps}$ [10]. MIMO uses multiple antennas for transmission and reception for transmission of the same data at phases. It defines different $\mathrm{M} X \mathrm{~N}$ configurations from $1 X 1$ to $4 X 4$ [5]. As the number of antennas for transmission and reception increases, the performance of the channel increases significantly. MIMO operates in two different modes: SDM and STBC.

- In Spatial Division Multiplexing (SDM), the output stream is divided into multiple sub streams which are then spatially multiplexed and transmitted from multiple antennas. At the receiving side, these streams are demultiplexed and recovered.

- In Space Time Block Coding (STBC), the same output stream is transmitted over multiple antennas simultaneously. At the receiving side, the multiple streams with different strength are compared to recover the original stream.

\begin{tabular}{|c|c|c|c|c|c|}
\hline $\mathbf{8 0 2 . 1 1}$ & $\begin{array}{c}\text { Freq } \\
(\mathbf{H z})\end{array}$ & $\begin{array}{c}\text { Through put } \\
(\mathbf{M b} / \mathbf{s})\end{array}$ & $\begin{array}{c}\text { Max Bit rate } \\
(\mathbf{M b} / \mathbf{s})\end{array}$ & Modulation & $\begin{array}{c}\mathbf{r}_{\text {in }} \\
(\mathbf{m})\end{array}$ \\
\hline $\mathbf{A}$ & 5 & 23 & 54 & OFDM & $\sim 35$ \\
\hline $\mathbf{B}$ & 2.4 & 4.3 & 11 & DSSS & $\sim 38$ \\
\hline $\mathbf{G}$ & 2.4 & 19 & 54 & OFDM & $\sim 38$ \\
\hline $\mathbf{N}$ & $2.4,5$ & 74 & 248 & OFDM & $\sim 70$ \\
\hline
\end{tabular}

Table 2.1- 802.11n standard specification [2]

\subsubsection{Channel bonding}

It uses a $40 \mathrm{MHz}$ channel which means greater bandwidth which ultimately leads to better performance in WLAN. The $40 \mathrm{MHz}$ channel [5] behaves like a combination of two $20 \mathrm{MHz}$ channel. It can also operate in two modes: $20 \mathrm{MHz}$ mode and $40 \mathrm{MHz}$ mode. The $20 \mathrm{MHz}$ [5] mode is used if the bandwidth availability is limited. The $40 \mathrm{MHz}$ channel provides two times more desirability than $20 \mathrm{MHz}$ channel.

\subsection{MAC layer enhancement}

Frame aggregation is a feature first introduced in 802.11e Wireless LAN where two or more frame is sent in a single transmission to increase the throughput. Frame aggregation reduces overhead since it transmits multiple frames as a single aggregate frame it reduces traffic 


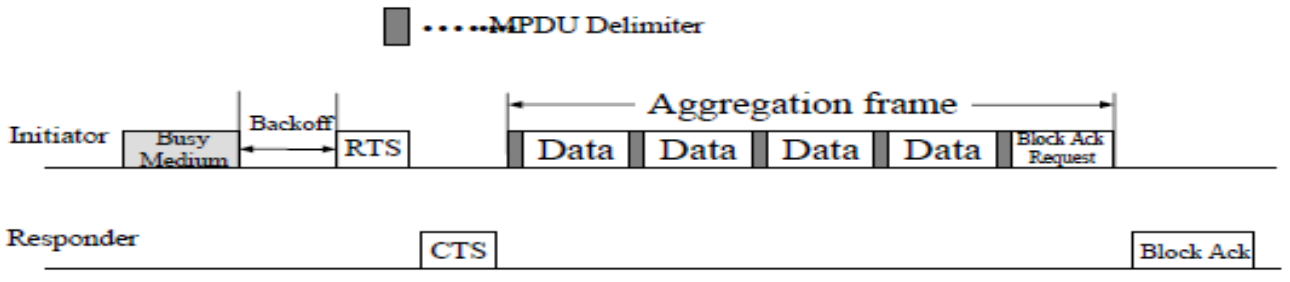

Fig 2.1- Frame Aggregation method [9]

There are three types of frame aggregation techniques- MSDU, MPDU and Two Level Frame Aggregation.

\subsubsection{MAC Service Data Unit (MSDU)}

This is the unit of transmission used at the MAC layer for data which is received from the upper layer. The data received from the logical link control (LLC). This LLC sub layer is present above the medium access control (MAC) sub layer in a protocol stack.

\subsubsection{Aggregated MAC Service Data Unit (A-MSDU)}

Multiple MAC service data unit are combined to form the payload of a MPDU packet. The entire aggregate packet will have a single frame header and these packets are destined to the same client and should have same access category. The main motivation of A-MSDU technique is the smaller size of Ethernet header when compared to 802.11 header. Hence multiple Ethernet frames can be combined to form A-MSDU. Since individual sub frames don't have frame checksum, selective retransmission of corrupted sub frames will not be possible. Sub frames will have same traffic identifier (TID) and sequence number [2]. For constructing an A-MSDU there is some limitations to follow such as, every MSDUs should have the same traffic identifier value. The sustained time of A-MSDU should be equal to the maximum sustained time of its constituent elements. In the sub frame header the values of destination address and sender address should match with the values of receiver address and the transmitter address in the MAC header.

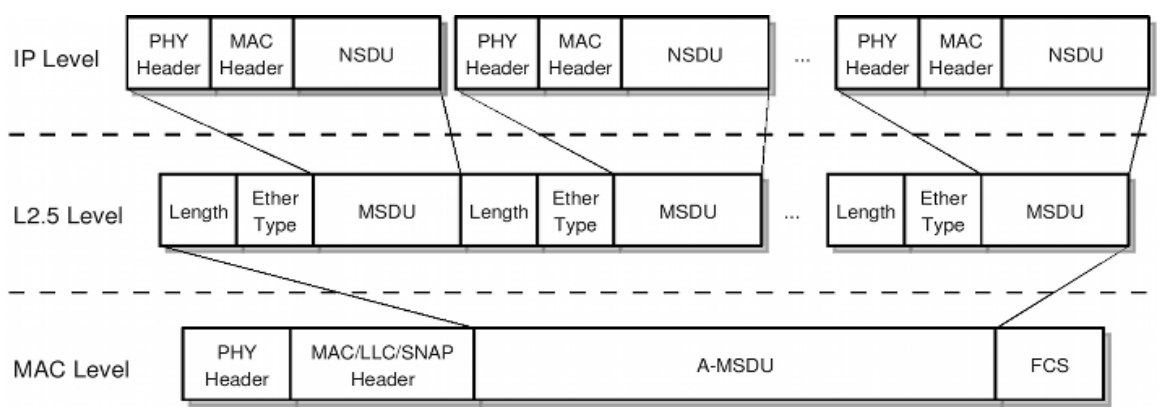

Fig 2.2- Format of A-MSDU [10]

The maximum frame length allowed in A-MSDU scheme is $3 \mathrm{~kb}$ or $7 \mathrm{~kb}$ [2]. Every sub frame consists of a header these headers is followed by packets that arrived from logical link control and $0-3$ bytes of padding [10]. The padding size depends on each sub frame but the last one must be a multiple of four bytes [9]. 


\subsubsection{MAC Protocol Data Unit (MPDU)}

MAC protocol data units are the frames passed from the MAC layers into the PHY layer. This MPDU mechanism is more efficient with high data rates because of block acknowledgement. This block acknowledgement allows each of the data aggregated frames to be acknowledged individually and if error occurred it is retransmitted.

\subsubsection{Aggregated MAC Protocol Data Unit (A-MPDU)}

Multiple MAC protocol data unit with common PHY header are aggregated as a single A-MPDU. The size of sub frames should be multiple of 4 bytes otherwise length is adjusted by adding padding bytes [9]. Each PDU in the frame has its known header and cyclic redundancy check so in case of transmission error it is sufficient to send only the lost PDU thereby improving the throughput [3]. A-MPDU functions after the encapsulation process of MAC header here the matching traffic identifier is not considered but all the MPDUs present within an A-MPDU should be addressed to the same receiver address. Since there is no holding time to form AMPDU the number of MPDUs to be aggregated depends on the packets that are already present in the transmission queue.

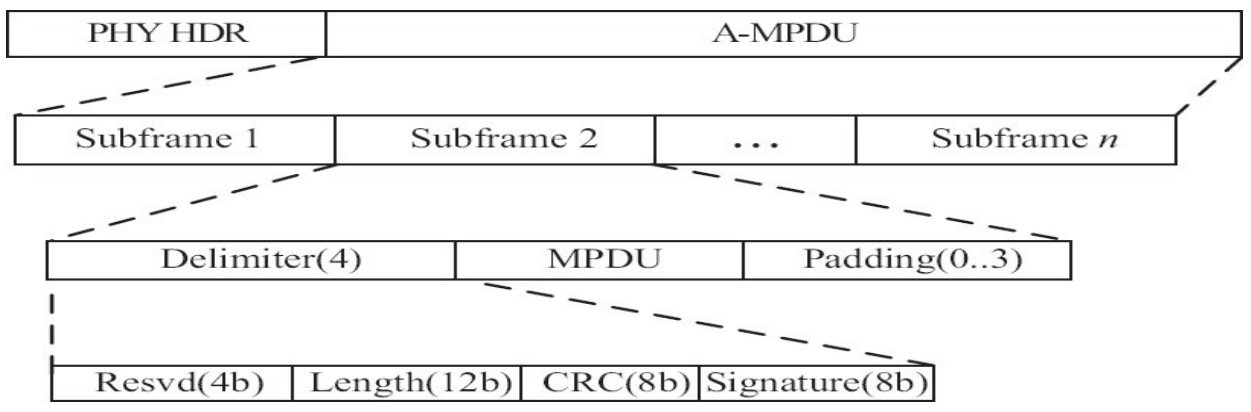

Fig 2.3- Format of A-MPDU [10]

Before MPDU a set of delimiters are added and in its tail padding bits varied from $0-3$ bytes are added to define the length and position inside the aggregated frame the delimiter header is used. For determining the structure of A-MPDU the usage of MPDU delimiters and PAD bytes are must. After the A-MPDU is received it checks for the MPDU delimiter for any errors in cyclic redundancy check. If the value is correct then the MPDU is extracted else it continues until it reaches the end of physical layer service data unit

\subsection{Block acknowledgement}

The block acknowledgement is a single acknowledgement frame that acknowledges multiple received frames.802.11n allows a receiver to cumulatively acknowledge multiple frames with a single Block Acknowledgement [9]. 
International Journal of Wireless \& Mobile Networks (IJWMN) Vol. 5, No. 6, December 2013

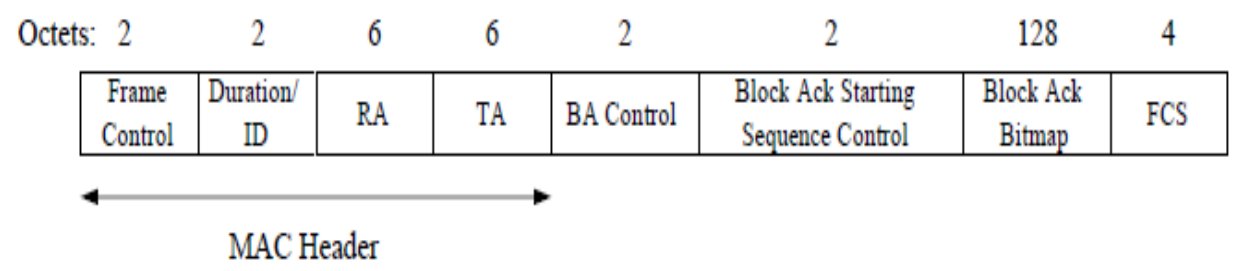

Fig 2.4 - Block acknowledgement format

To confirm the frame delivery block acknowledgment minimizes the number of ACKs that a receiver must send to a transmitter.

\begin{tabular}{|c|c|c|}
\hline Access Category & Frame Aggregation & Reasons \\
\hline Voice & NA & $\begin{array}{c}\text { Voice packets are delay- } \\
\text { sensitive. These packets need } \\
\text { strict latency, jitter and } \\
\text { bandwidth requirement [8] } \\
\text { use of aggregation degrade } \\
\text { the performance of voice } \\
\text { packets since these has to wait } \\
\text { for other packets. }\end{array}$ \\
\hline Video & A-MPDU & $\begin{array}{c}\text { Video packets have strict } \\
\text { bandwidth demand but loser } \\
\text { latency in jitter demand [8]. } \\
\text { Video packets have high data } \\
\text { rate so waiting for other } \\
\text { packets exceed the maximum } \\
\text { tolerable delay [7]. }\end{array}$ \\
& & $\begin{array}{c}\text { Delay insensitive and no } \\
\text { bandwidth requirements [8] } \\
\text { making the packets of this } \\
\text { access category will not have } \\
\text { any impact on their } \\
\text { performance. }\end{array}$ \\
\hline
\end{tabular}

Table 2.2 -Selecting required Aggregation Scheme

\section{MOTIVATION FOR THE PROPOSED WORK (Issues in frame aggregation)}

Generic frame aggregation cannot be applied for all access categories because of different QoS requirements [8]. Frame aggregation is unable to function effectively in unsaturated traffic. As one side, the sender has to wait for more frames which deliberately increases the latency there by affecting delay sensitive packets. Other choice is, it is forced to send the available packet which does not use the bandwidth efficiently. In the above cases, the advantage gained by the frame aggregation is lost. 
International Journal of Wireless \& Mobile Networks (IJWMN) Vol. 5, No. 6, December 2013

\section{PROPOSED WORK}

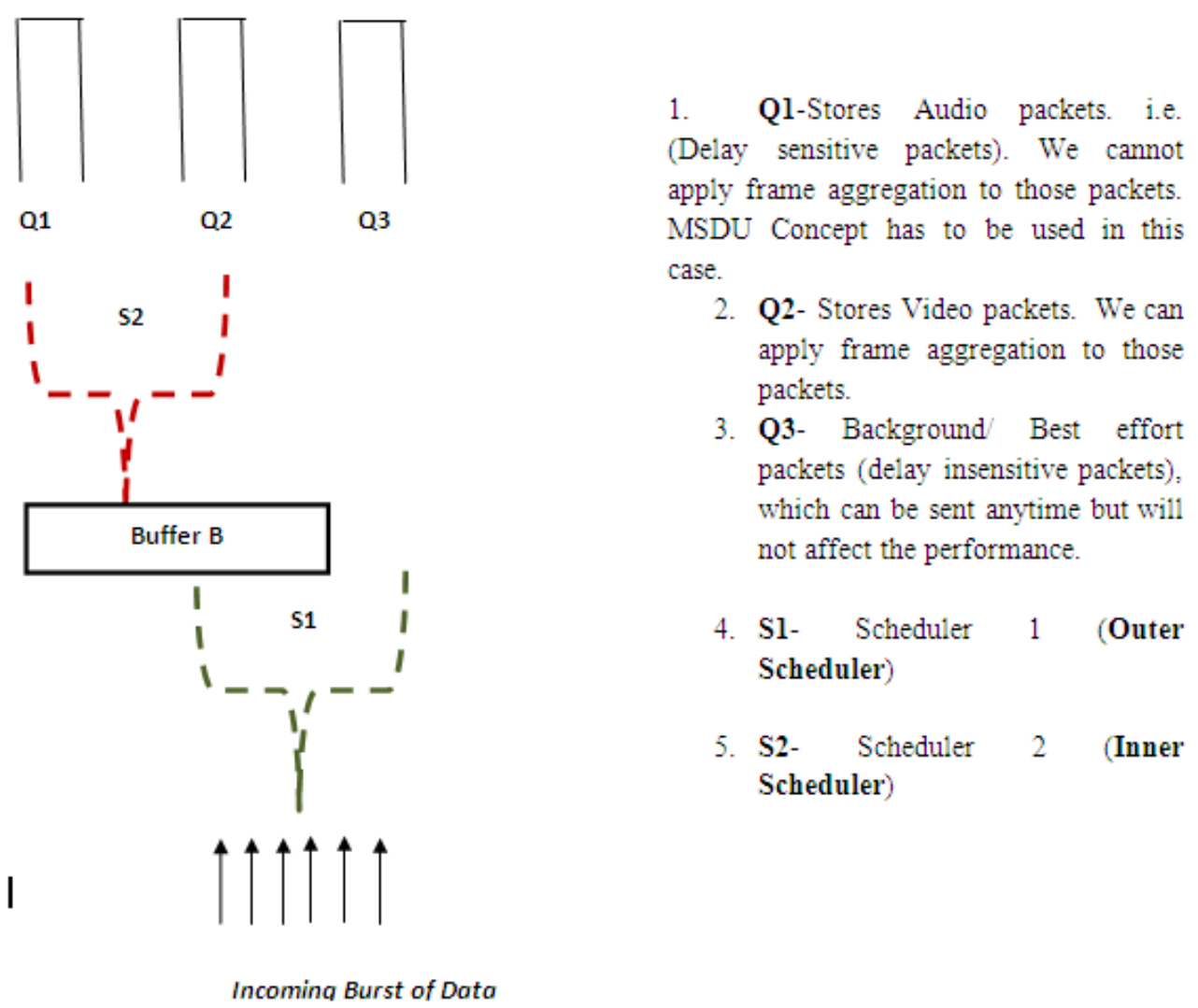

Fig 4.1- Bi-Scheduler Implementation Using Queues

\subsection{Working of Bi-Scheduler Technique for Throughput improvement in Frame Aggregation}

The idea is based on the fact that since Audio packets are delay sensitive and have low data rate, so making them wait in the queue will introduce lot of delay in network and degrades its performance. Thus, A-MSDU technique must be applied to such packets in order to increase their throughput. Hence frame aggregation (A-MSDU) can be effectively applied only to improve the throughput of high data rate packets. Therefore, making such types of packets wait in the queue (to attain the desired size for aggregation) does not significantly affect the throughput of the network. While Background and Best Effort packets are delay insensitive and making them wait will not have any impact in the through put of the network.

1. Initially, the Outer Scheduler (S1) separates the delay sensitive frames i.e. Voice/ Audio frames from the incoming burst of multipart data based on their Access Category (AC). These frames are directly enqued into Queue Q1, where they wait for the other frames of same AC to arrive in order to form A-MSDU. A timer is also maintained separately for this queue in order to minimize the delay that is deliberately introduced in this process. If the timer for this queue expires, automatically the contents of Q1 will be sent to respective destination irrespective of the number of frames available at that time. 
International Journal of Wireless \& Mobile Networks (IJWMN) Vol. 5, No. 6, December 2013

2. The remaining frames i.e. Video and Background/ Best-Efforts are stored temporarily in Buffer B1 of Inner Scheduler (S2). S2 then separates Video frames from Best-Efforts frames and enques them in queue Q2, where they are aggregated to form A-MPDU packets. The rest of the frames are enqued in Q3. A common timer is also maintained for Q2 and Q3.

a. When Q2 has reached the aggregated size before the timer expires, it's transferred to the respective destination.

b. If Q2 has not reached the aggregated size before the timer expires, then that many remaining packets from Q3 are taken to attain the desired size and sent to the destination.

3. If, simultaneously both Q1 and Q2 have reached the desired size before the timer expires, then our algorithm gives preference to Audio packets i.e. contents of Q1 will be sent first, since they are highly delay sensitive packets. After, resetting Q1's timer, Q2 contents will be sent to the respective destination.

Fig 4.2 Bi-Scheduler Algorithm's Flowchart

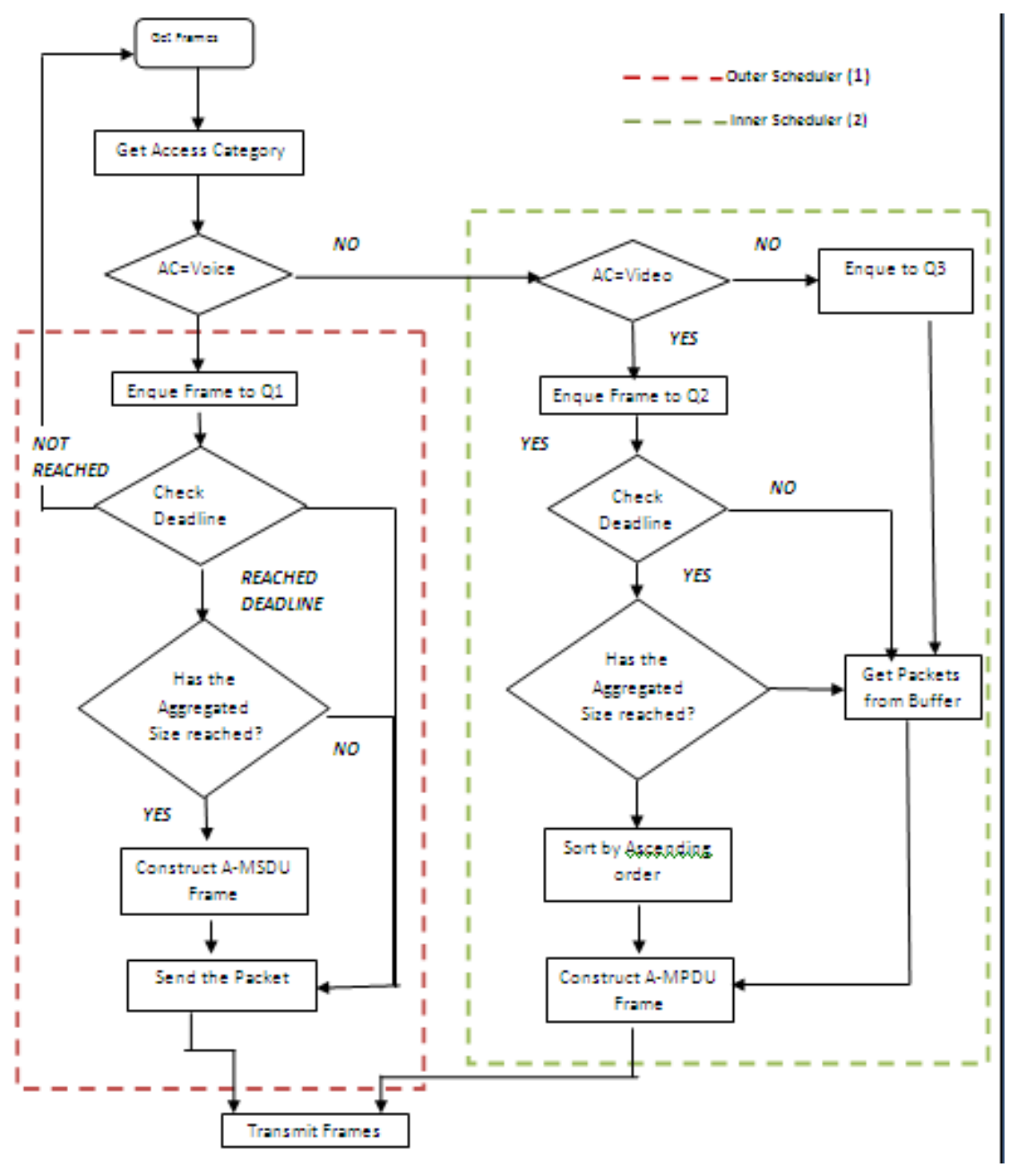




\section{CONCLUSION}

The research work proposes a new Bi-Scheduler Mechanism to improve the throughput of IEEE 802.11n WLAN using efficient frame aggregation technique. Depending on the Access Category (AC), the proposed scheduler decides whether to implement A-MSDU or A-MPDU mechanism, in order to efficiently transmit the frames to the destination. The future work will be to simulate and experimentally verify the proposed scheduler mechanism effectively.

\section{ACKNOWLEDGEMENTS}

We would like to acknowledge and thank the Defence Research Development Organisation (DRDO), New Delhi, India for granting us extra mural research funds for carrying out the research work is a part of the research project titled "Network Throughput Enhancement in distributed using Cross layer optimisation".

We would also like to thank the Head of the Department of Department of CSE and other faculty members who gave us necessary support to implement this project.

\section{REFERENCES}

[1] Nasreddine Hajlaoui, Issam Jabri and Maher Ben Jemaa, "Experimental Performance Evaluation and Frame Aggregation Enhancement in IEEE 802.11n WLANs".

[2] J.Kolap, S.Krishnan, N.Shala,"Frame Aggregation Mechanism for High Throughputin 802.11n WLANS".

[3] Selvam T, Srikanth.S, “A Frame Aggregation Scheduler for IEEE 802.11n”.

[4] WI-FI Certified TM 802.11n Draft2.0.

[5] L.Kriara, M.K. Marina, A.Farshad, "Characterization of 802.11n Wireless LAN performance via Test bed measurement and statistical Analysis".

[6] A.Saif, M.othman, S.Subramaniam and N.A.A. Hamid "Frame Aggregation in Wireless Techniques and Issues".

[7] S.Gubner and C. Luidemann, "Evaluating the Impact of Frame Aggregation on Video Streaming over IEEE802.11n Multi hop Network".

[8] B.Selvig, A.Bai, T.Skeie, P. Engelstad, "Differentiated Service in 802.11e WLANS"

[9] T.Nakajima, Y.Utsuomiya, Y. Nishibayashi, T.Tandai, T.Adachi and M. Takagi "Compressed Block Acknowledgment - an efficient selective repeat mechanism for IEEE 802.11n"

[10] Sources are also taken from Google and Wikipedia.

\section{Authors}

\section{Ms Vanaja Ramaswamy}

Assistant professor, Department of Computer Science and Engineering, Sri Venkateswa ra college of Engineering, Sriperumpudur Taluk, Tamilnadu,IndiaCo-Principal Investigator of DRDO Extra mural Research Project.Area of Interest : Operating System, Data structures and Computer Networks

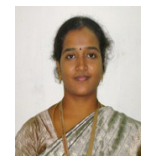

\section{Hamsalekha Venkatesh}

Final Year Student (B.E.), Department of Computer Science and Engineering Sri Venkateswara College Of Engineering. Sriperumpudur Taluk, Tamilnadu, India,Area of Interest: Computer Networks an d Graph Theory.

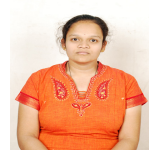


International Journal of Wireless \& Mobile Networks (IJWMN) Vol. 5, No. 6, December 2013

\section{Bhargavi Sridharan}

Final Year Student (B.E.), Department of Computer Science and Engineering S ri Venkateswara College Of Engineering. Sriperumpudur Taluk, Tamilnadu, India,Area of Interest: Computer Networks and object oriented programming.

\section{Abinaya sivarasu}

Final Year Student (B.E.), Department of Computer Science and Engineering Sri Venkateswara College Of Engineering. Sriperumpudur Taluk, Tamilnadu, India,Area of Interest: Computer Networks and operating system
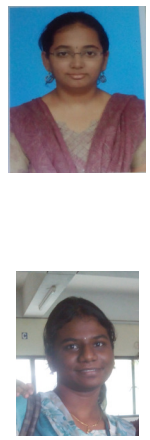